\title{
Radiative corrections and Lorentz violation
}

\author{
A. F. Ferrari ${ }^{1,2, a}$, J. R. Nascimento ${ }^{3, b}$, A. Yu. Petrov ${ }^{3, c}$ \\ ${ }^{1}$ Universidade Federal do ABC, Centro de Ciências Naturais e Humanas, Rua Santa Adélia, 166, Santo André, SP 09210-170, Brazil \\ ${ }^{2}$ Indiana University Center for Spacetime Symmetries, Indiana University, Bloomington, IN 47405-7105, USA \\ ${ }^{3}$ Departamento de Física, Universidade Federal da Paraíba, Caixa Postal 5008, 58051-970 João Pessoa, Paraíba, Brazil
}

Received: 22 November 2019 / Accepted: 2 May 2020 / Published online: 22 May 2020

(C) The Author(s) 2020

\begin{abstract}
Radiative corrections in Lorentz violating (LV) models have already received a lot of attention in the literature in recent years, with many instances where a $L V$ operator in one sector of the Standard Model Extension (SME) generates, via loop corrections, one of the LV coefficients in the photon sector, which is probably the most understood and well constrained part of the SME. In many of these works, however, the now standard notation of the SME is not used, which can obscure the comparison of different results, and their possible phenomenological relevance. In this work, we fill this gap, trying to build up a more general perspective on the topic, bringing many of the results to the SME conventional notation and commenting on their possible phenomenological relevance. We uncover one example where a result already presented in the literature can be used to place a stronger bound on the temporal component of the $b_{\mu}$ coefficient of the fermion sector of the SME.
\end{abstract}

\section{Introduction}

The idea that Lorentz symmetry might be violated by new physics at the Planck scale is one of the motivations for the development of the Standard Model Extension (SME) [1,2] as an effective field theory, based on the internal symmetries and field content of the Standard Model, and incorporating a general set of Lorentz violating operators. In the minimal SME, power counting renormalizability is enforced, so only operators with mass dimensions four or less are included, while the non-minimal extension of the SME includes all operators with higher mass dimensions [3-7], thus representing the most general description of low-energy Lorentz violating effects originating from new physics at some very

\footnotetext{
a e-mail: alysson.ferrari@ufabc.edu.br (corresponding author)

b e-mail: jroberto@fisica.ufpb.br

${ }^{c}$ e-mail: petrov@ fisica.ufpb.br
}

high energy scale. Lorentz symmetry being one of the cornerstones of Quantum Field Theory (QFT) as we know it, Lorentz violation (LV) brings with it many interesting theoretical questions. At the same time, a fruitful experimental program have been using the SME framework to obtain new and improved tests of Lorentz invariance from many experiments and astrophysical observations $[8,9]$.

The source of LV in the SME is generally assumed to be new physics at some high energy scale, for example spontaneous symmetry breaking in a more fundamental theory such as string theory [10], in which case the constant tensors that couple to the LV operators arise as vacuum expectation values of tensor fields in this theory. One may also consider explicit breaking, which amounts to assume some unknown mechanism generating $\mathrm{LV}$ at the fundamental level, and therefore the LV background tensors are taken to assume, unspecified non-zero values. In the non gravitational sector of the SME, the difference between explicit and spontaneous LV breaking is not essential in principle, but in the gravitational sector, one finds that explicit breaking is in general incompatible with the usual geometric picture of general relativity [11]. In this work, we will be mostly interested in the non-gravitational sector, so LV can be assumed to be explicit for simplicity.

The photon sector of the SME is probably its most well studied part, being of utmost importance from the phenomenological viewpoint since the most stringent constraints on Lorentz violation are generally obtained by studying LV effects on photon propagation. It is described by the Lagrangian density

$$
\begin{aligned}
\mathcal{L}_{\text {photon }}= & -\frac{1}{4} F^{\mu \nu} F_{\mu \nu}+\frac{1}{2} \epsilon^{\kappa \lambda \mu \nu} A_{\lambda}\left(\hat{k}_{A F}\right)_{\kappa} F_{\mu \nu} \\
& -\frac{1}{4} F_{\kappa \lambda}\left(\hat{k}_{F}\right)^{\kappa \lambda \mu \nu} F_{\mu \nu},
\end{aligned}
$$


where

$$
\begin{aligned}
& \left(\hat{k}_{A F}\right)_{\kappa}=\sum_{d \text { odd }}\left(\hat{k}_{A F}^{(d)}\right)_{\kappa}^{\alpha_{1} \cdots \alpha_{(d-3)}} \partial_{\alpha_{1}} \cdots \partial_{\alpha_{(d-3)}}, \\
& \left(\hat{k}_{F}\right)^{\kappa \lambda \mu \nu}=\sum_{d \text { even }}\left(\hat{k}_{F}^{(d)}\right)^{\kappa \lambda \mu \nu \alpha_{1} \cdots \alpha_{(d-4)}} \partial_{\alpha_{1}} \cdots \partial_{\alpha_{(d-4)}},
\end{aligned}
$$

$d \geq 3$ being the dimension of the corresponding operator (for more details see [3]). The minimal photon sector of the SME is obtained by the restriction to $d=3$ and $d=4$ for the CPT-odd and CPT-even terms, corresponding to the original $k_{A F}$ and $k_{F}$ defined in [2]. Some of the fundamental references regarding this sector of the SME are [12-15], and the most recent experimental limits can be found in [8]. It is noteworthy to recall that the $k_{A F}$ coefficient, and parts of the $k_{F}$, induce birefringence in the vacuum, which leads to very strong experimental constraints, from astrophysics, of order $10^{-43} \mathrm{GeV}$ for $k_{A F}$, and $10^{-37}$ for the birefringent components of $k_{F}$.

From the theoretical viewpoint, the quantum impacts of the LV operators in the SME already received much attention. Lorentz symmetry being so essential do quantum field theory, whether the full renormalization program can be carried out consistently for the minimal SME, which is power counting renormalizable, is an interesting question, which has been positively answered for the QED [16], electroweak [17], scalar and Yukawa [18] sectors. Interesting results have also been obtained regarding the Källén-Lehman representation [19] and the properties of asymptotic states [20] due to Lorentz violation, showing that much of the structure of QFT survives the introduction of LV as done in the SME, still allowing for nontrivial modifications.

Even from the phenomenological point of view, the study of quantum corrections might be of interest, since they can connect LV coefficients in different sectors of the framework, thus making it possible to transfer bounds found in one sector to the other. The general idea can be explained by recalling the first example of this mechanism [2,21-24], where the integration over a fermion loop, including a LV operator involving an axial vector $b^{\mu}$, was shown to generate a finite correction to the photon effective action, proportional to the well known Carroll-Field-Jackiw (CFJ) term [12]. Strong experimental bounds on the CFJ coefficient were already derived in [12], and theoretical consistency of the mechanism presented in [24] would allow one to translate this bounds to the original $b^{\mu}$ coefficient. However, from the start it was recognized that the generated CFJ term was finite, yet ambiguous, its value depending on the regularization scheme, thus being one example of "finite but undetermined" quantum corrections [25]. Several different approaches have been developed to understand this ambiguity (see for example [26-28] and references therein), and the conclusions seems to be that, in this particular case, gauge invariance enforces the generated
CFJ term to vanish. In any case, this very first example in the study of quantum corrections induced by the LV coefficients of the SME already shows the complexity and the potential for interesting theoretical and phenomenological discussions related to this matter.

These investigations motivated many other, where specific LV operators where generated as radiative corrections. Restriction to the minimal SME allows for more systematical studies such as the ones presented in [16], while the non-minimal SME can only be understood as an effective field theory. The case of finite and non ambiguous corrections is particularly interesting since it could relate LV couplings from different sectors of the framework, and present itself as a consistent way to generate LV from some more " fundamental " setup (assuming for instance the integrated field not to be one of the fields in the Standard Model). When divergent corrections to a given LV operator are generated, this operator has to be introduced from the very beginning to act as a counterterm to cancel this divergence, leaving behind an arbitrary finite constant that has to be fixed by some physical condition. Even so, this approach have been used in literature to infer bounds on LV coefficients, either by implicitly assuming all finite constants to be of order one, or by fixing it using minimal subtraction $[29,30]$.

In this work, we put in a more general and systematic perspective the problem of radiative corrections in the SME, focusing mostly on its potential to provide new, indirect, bounds on LV coefficients. We will fill some gaps in the literature, in the sense of presenting the results in the standard SME notation, which greatly facilitates the comparison with experimental results. In doing so, we will show that new and interesting results can be obtained. We uncover an example where a result already obtained in the literature, regarding higher orders corrections induced by the axial vector $b^{\mu}$, can actually provide better constraints than the ones already known for one component of the $b^{\mu}$ coefficient. This result is surprising, since the relevant correction is second order in $b^{\mu}$, as well as (being a quantum correction) suppressed by powers of the coupling constant. We believe this example points to an interesting direction, where many other indirect bounds could be placed, from the study of loop corrections in $\mathrm{LV}$ theories.

The technique employed within this paper in order to obtain Lorentz-breaking contributions to the effective Lagrangians has been used in [24] and afterwards, in many other papers, is as follows. We suggest some new couplings, involving Lorentz-breaking constant vectors or tensors, between spinor and vector (or scalar) fields, and calculate the contributions to effective action proportional to lower orders of these Lorentz-breaking vectors (tensors). In many cases, this approach is equivalent to expanding the fermionic determinant in the Lorentz-breaking extension of a theory up to lower orders in Lorentz-breaking parameters. Throughout 
this paper, we apply this technique to various extensions of QED and Yukawa model.

The structure of the paper looks like follows. In Sect. 2 , we discuss the radiative corrections generated by the $b$ coefficient only. In Sect. 3, we look at the minimal interactions between gauge and spinor fields, and the corresponding operators generated in the gauge sector, extending this study for the non-minimal QED extension in Sect.4. In Sect.5, we carry the same study for scalar-spinor couplings, and in Sect. 6 for the contributions with external spinors. Finally, Sect. 7 contains our conclusions and final remarks.

\section{Radiative corrections: the case of the $b$ coefficient}

Maybe the most studied instance of the mechanism we are interested in, already mentioned in the introduction, is the one involving the axial vector $b_{\mu}$, mainly due to the fact that the relevant calculation is puzzled by an ambiguity. Specifically, we are interested in the corrections induced in the photon sector from the LV minimal operator

$V_{b}=b_{\mu} \bar{\psi} \gamma^{\mu} \gamma_{5} \psi$

This LV insertion in a fermion loop contributing to the twopoint photon vertex function generates the CFJ term [2,24],

$\mathcal{L}_{e f f} \supset C_{0} e^{2} \epsilon^{\mu \nu \lambda \rho} b_{\mu} A_{\nu} \partial_{\lambda} A_{\rho}$,

$e$ being the electric charge. This amounts, in the SME notation, to the generation of a minimal $k_{A F}$ term with $k_{A F} \sim b$. In this result, however, $C_{0}$ is a finite and ambiguous constant. The ambiguity is no surprise since it comes from a triangular fermion loop with one insertion of the LV twofermion vertex $b_{\mu} \gamma^{\mu} \gamma_{5}$, whose corresponding amplitude is therefore quite similar to the well known anomalous triangle diagram in the Standard Model, where one of the external photon lines is taken at zero external momenta: $A(p)_{\mu} \gamma^{\mu} \gamma_{5} \rightarrow A(0)_{\mu} \gamma^{\mu} \gamma_{5}$. It has also been shown that in the non-Abelian case, for $N>1$ spinor fields, the $N$-fields generalization of the $V_{b}$ operator generates the non-Abelian extension of the CFJ term [31],

$\mathcal{L}_{e f f} \supset C_{0} \epsilon^{\mu \nu \lambda \rho} b_{\mu} \operatorname{tr}\left(g^{2} A_{\nu} \partial_{\lambda} A_{\rho}+\frac{2 g^{3}}{3} A_{\nu} A_{\lambda} A_{\rho}\right)$,

$C_{0}$ being the same ambiguous constant. Several works in the literature claim that gauge invariance actually enforces $C_{0}=0[28,32,33]$, so this mechanism can hardly be argued to generate in a consistent way the minimal $k_{A F}$ term in the photon sector of the SME.

It proves interesting to study higher order corrections derived from the $V_{b}$ insertion, both in the sense of an expansion in derivatives of the electromagnetic field, as well as higher orders in the LV coefficient. In the first sense, keeping only one $V_{b}$ insertion, but calculating higher derivative contributions, the result yields the higher-derivative CFJ-like term

$\mathcal{L}_{e f f} \supset C_{1} \frac{e^{2}}{m^{2}} \epsilon^{\beta \mu \nu \rho} b_{\beta} A_{\mu} \square F_{v \rho}$,

with $C_{1}=1 / 24 \pi^{2}$ being a finite, well defined constant [34]. In the SME notation, this amounts to the generation of a dimension five coefficient

$\left(\hat{k}_{A F}^{(5)}\right)_{\kappa}^{\alpha \beta}=C_{1} \frac{e^{2}}{2 m^{2}} b_{\kappa} \eta^{\alpha \beta}$.

The interesting aspect of this calculation is that the result is ambiguity-free, so one could hope to infer experimental bounds on $b$ from the constraints on the photon sector coefficient $\hat{k}_{A F}^{(5)}$. However, at the moment, experimental constraints on dimension five photon coefficients are obtained only from the study of free propagation of photons [8], which means that leading $L V$ effects may be obtained by imposing the usual dispersion relation $\eta_{\mu \nu} p^{\mu} p^{\nu}=0$ in the general expressions for $\hat{k}_{A F}$ given in Eq. (2). This, together with Eq. (7), means that the LV operator in Eq. (6) does not contribute to wave propagation. As a conclusion, in this example, even if the generated LV operator is finite and free of ambiguities, its particular form is such that no experimental constraints can be inferred from this result at the present.

Now considering corrections at second order in $b, V_{b}$ can contribute to the CPT-even, minimal, $k_{F}$ coefficient. Considering two $V_{b}$ insertions, one indeed obtains the aether term,

$\mathcal{L}_{e f f} \supset-C_{2} \frac{e^{2}}{m^{2}} b^{\mu} b_{\lambda} F_{\mu \nu} F^{\lambda \nu}$,

with $C_{2}=1 / 6 \pi^{2}$. This result is finite by power counting and therefore ambiguity-free $[35,36]$, and corresponds to the SME minimal coefficient

$$
\begin{aligned}
\left(k_{F}^{(4)}\right)^{\mu \nu \alpha \beta}= & -C_{2} \frac{e^{2}}{m^{2}}\left(b^{\mu} b^{\alpha} \eta^{\nu \beta}-b^{\nu} b^{\alpha} \eta^{\mu \beta}\right. \\
& \left.-b^{\mu} b^{\beta} \eta^{\nu \alpha}+b^{\nu} b^{\beta} \eta^{\mu \alpha}\right) .
\end{aligned}
$$

We note that any charged particle with non-zero $b_{T}$ yields non-zero contributions into $\left(k_{F}^{(4)}\right)^{\mu \nu \alpha \beta}$.

This is a well defined quantum correction and, despite being of second order in $b$, as well as suppressed by a power of $e^{2}$, it provides competitive constraints on some of the $b_{\mu}$ coefficients, given that birefringent components of $k_{F}$ are strongly constrained at the order $10^{-37}$ due to birefringence effects in gamma ray bursts [37]. Therefore, from Eq. (9) we could expect components of $b$ to be limited by $b^{2}<6 \pi^{2} \mathrm{~m}^{2} / e^{2} \times 10^{-37}$, amounting to $|b|<3 \times 10^{-15} \mathrm{GeV}$ for protons and $|b|<1.5 \times 10^{-20} \mathrm{GeV}$ for electrons, for example. We have to compare these with the current experimental limits for the $b$ coefficient, which are always quoted in the Sun centered frame, taken as a standard frame for the reporting of experimental bounds on LV coefficients [8]. As 
of 2019, we verify these bounds suggested by the radiative corrections are not better than the ones already known for the spatial components of $b^{\mu}$ for protons and electrons, but they are better than the ones obtained for the temporal components of $b_{\mu}$, which are of order $\left|\left(b_{T}\right)_{p}\right|<7 \times 10^{-8} \mathrm{GeV}$ for protons and $\left|\left(b_{T}\right)_{e}\right|<10^{-15} \mathrm{GeV}$ for electrons [8], where the $T$ subscript is the standard notation for the temporal component of a vector in the Sun centered frame.

For the sake of clarity, we can simply assume that $b^{\mu}=\left(b_{T}, \overrightarrow{0}\right)$ in the Sun centered frame, and we take into account that quantum corrections will induce a corresponding $\left(k_{F}\right)^{\mu \nu \alpha \beta}$ of the form given in Eq. (9). One simple parametrization for the components of $k_{F}$ relevant for birefringence is in terms of the ten $k^{a}$ coefficients defined in [13], and it is easy to verify that we generate in this way non-vanishing birefringent coefficients $k^{a}=C_{2} e^{2} b_{T}^{2} / m^{2}$ for $a=3,4$, which are subjected to the aforementioned $10^{-37}$ constraints. In summary, we conclude that the consistency of the quantum corrections given in Eq. (8) imply in the following constraint for the temporal component of the $b_{\mu}$,

$\left|b_{T}\right|<\pi m / e \sqrt{6 \times 10^{-37}}$,

which is established in an indirect way, from the observational constraint on $k_{F}$ reported in [37]. This implies in new stronger constraints on $b_{T}$ coefficients, for example

$\left|\left(b_{T}\right)_{p}\right|<3 \times 10^{-15} \mathrm{GeV}$

for protons and

$\left|\left(b_{T}\right)_{e}\right|<1.5 \times 10^{-20} \mathrm{GeV}$

for electrons.

One can look for even higher orders corrections in $V_{b}$, even if these are not expected to lead to competitive bounds. Considering three $V_{b}$ insertions, one obtains a linear combination of the higher-derivative CFJ-like term (6) and the Myers-Pospelov term [38]

$\mathcal{L}_{e f f} \supset \frac{e^{3}}{m^{4}} C_{3} b^{\alpha} F_{\alpha \mu}(b \cdot \partial) \epsilon^{\beta \mu \nu \rho} b_{\beta} F_{\nu \rho}$,

where $C_{3}$ is also an ambiguity-free constant [39]. This model can be obtained from the general SME formalism by a specific choice of $\hat{k}_{A F}^{(5)}$, corresponding to a particular isotropic limit of Lorentz violation, leading to modified dispersion relations for photons (which were the original motivation for the introduction of the model in [38]), see section IV-F in [3] for more details. For more insertions, it is natural to expect the appearance of terms including fourth and higher orders in derivatives, meaning contributions to $k_{F}^{(d)}$ for $d \geq 6$. Up to now, apart from the general discussion in [3], further consequences of these dimension six terms have only been studied at the tree level [40].
Despite so much work have been devoted to the radiative corrections induced by the $b^{\mu}$ coefficient, the phenomenological implications unveiled in this section have not been extensively taken into account as far as we know of. In particular, we stress the unexpected possibility of using higher order LV corrections to infer competitive (but indirect) bounds on some weakly bounded LV coefficients, provide we can relate this with finite and well defined quantum corrections to the strongly constrained $k_{A F}$ and/or $k_{F}$ coefficients.

\section{The Minimal QED extension}

The most generic Lorentz-breaking extension of QED containing only terms of renormalizable dimensions, also called the minimal QED extension, is given by the following Lagrangian [2],

$\mathcal{L}=\bar{\psi}\left(i \Gamma^{v} D_{v}-M\right) \psi+\mathcal{L}_{\text {photon }}^{(3,4)}$,

where

$\Gamma^{v}=\gamma^{v}+c^{\mu \nu} \gamma_{\mu}+d^{\mu \nu} \gamma_{\mu} \gamma_{5}+e^{v}+i f^{v} \gamma_{5}+\frac{1}{2} g^{\lambda \mu \nu} \sigma_{\lambda \mu}$

$M=m+a_{\mu} \gamma^{\mu}+b_{\mu} \gamma^{\mu} \gamma_{5}+\frac{1}{2} H^{\mu \nu} \sigma_{\mu \nu}$,

$D_{\mu}=\partial_{\mu}-i e A_{\mu}$ is the usual $U$ (1) covariant derivative, $\mathcal{L}_{\text {photon }}^{(3,4)}$ is the restriction of the Lagrangian in Eq. (1) to minimal (dimension three and four) operators, and $a^{\mu}, b^{\mu}, c^{\mu \nu}$, $d^{\mu \nu}, e^{\mu}, f^{\mu}, g^{\lambda \mu \nu}, H^{\mu \nu}$ are constant (pseudo)tensors, collectively known as the LV coefficients of the QED sector of the minimal SME, together with the $\kappa_{F}^{(4)}$ and $k_{A F}^{(3)}$ defined by Eq. (2).

While generality motivates Eq. (14), one should be aware that some of the LV couplings present in it might not be relevant to physics in most cases. The vector $a_{\mu}$ can be eliminated in a single fermion theory by a field redefinition $\psi \rightarrow e^{-i a \cdot x} \chi$, so it is usually disregarded (the situation changes when gravity is taken into account, however [11]). The antisymmetric part of the $c^{\mu \nu}$ coefficient can be removed by a redefinition of the gamma matrices, so $c^{\mu \nu}$ is usually taken to be symmetric; also, the antisymmetric part of $d^{\mu \nu}$, the trace and totally antisymmetric parts of the $g^{\lambda \mu \nu}$ terms are not expected to generate physical effects [2]. The $f^{\mu}$ coefficient can be shown to generate effects that can be exactly mimicked by the symmetric part of $c^{\mu \nu}$ [41], so it is also usually disregarded. A detailed discussion of the removal of spurious LV operators via field redefinitions can be found in [42] (see also [43] for a related discussion on the $b^{\mu}$ coefficient, and [44] for singular spinor fields and torsion). 
The photon sector of the SME being so well understood theoretically, and so well constrained by experiments, makes particularly interesting the study of the structure of quantum corrections that can be induced from different LV couplings in this specific sector of the SME. For the remainder of this section, we restrict ourselves to the original couplings being present in the minimal QED extension, as described in (14) (notice, however, that in several instances, the generated LV operators are themselves non-minimal), and discuss the most interesting cases of radiative corrections.

The quantum consequences of the $b$ coefficient in Eq. (15b) have already been discussed in the previous section, so we will consider hereafter the other relevant minimal couplings. We start with the operator proportional to the tensor $c_{\mu \nu}$,

$V_{c}=i c^{\mu \nu} \bar{\psi} \gamma_{\mu}\left(\partial_{v}-i e A_{v}\right) \psi$,

where $c^{\mu \nu}$ is taken to be symmetric. A minimal scenario including only the $c^{\mu \nu}$ coefficient was discussed in [45], where it was shown that the presence of the $c^{\mu \nu}$ coefficient as the only LV in the model does not modify the usual picture of the Adler-Bell-Jackiw anomaly and index theorem (for a more general discussion regarding the effects of LV in the discussion of anomalies in chiral gauge theories, see [46]). As for the radiative generation of corrections in the photon sector, calculations have been performed only considering a particular form of $c_{\mu \nu}$, parametrized by a constant vector $u_{\mu}$,

$c_{\mu \nu}=u_{\mu} u_{v}-\frac{\zeta}{4} \eta_{\mu \nu} u^{2}$,

so that, at $\zeta=0$ we have the simplest form $c_{\mu \nu}=u_{\mu} u_{\nu}$, and at $\zeta=1$ the $c_{\mu \nu}$ is traceless. Respecting CPT invariance, quantum corrections involving the $V_{c}$ insertion will contribute to the CPT-even photon coefficient $k_{F}$, starting at the first order, and the explicit form of this contribution involving up to three $c_{\mu \nu}$ insertions has been calculated in [47]. The most interesting case to quote is the first order in the traceless $c_{\mu \nu}$, where the leading (divergent) corrections generate the simple term

$\mathcal{L}_{e f f} \supset \frac{e^{2}}{2 \pi^{2} \epsilon} c_{\mu \rho} \eta_{\nu \sigma} F^{\mu \nu} F^{\sigma \rho}$,

corresponding to

$\left(k_{F}\right)_{\mu \nu \rho \sigma} \sim c_{\mu \rho} \eta_{\nu \sigma}-c_{\nu \rho} \eta_{\mu \sigma}-c_{\mu \sigma} \eta_{\nu \rho}+c_{\nu \sigma} \eta_{\mu \rho}$.

For $\zeta=0$, one obtains in particular the aether-like photon coefficient in Eq. (9), together with a rescaled Maxwell term proportional to $u^{2} F^{\mu \nu} F_{\mu \nu}$. However, unlike in the results generated from the CPT-odd couplings [35,36], in the case of the $c_{\mu \nu}$ insertions the aether term logarithmically diverges. Nevertheless, it should be noted that this aether-like divergent contribution disappears after a proper redefinition of fields and derivatives introduced in [16], so, afterwards, only the renormalization of the Maxwell term persists.
One more interesting example of study regarding the operator (16) is presented in [48], where the particular case of $c_{\mu \nu}$ characterized by only one constant $c_{e}$, with $\left(k_{F}\right)_{\mu \nu \rho \sigma}$ also described by a constant $c_{\gamma}$, is considered, and in this case the two-point function of the photon is obtained explicitly in all orders in $c_{e}, c_{\gamma}$, displaying a logarithmic dependence on $c_{e}-c_{\gamma}$.

Early works concerning radiative corrections generated from the term proportional to the coefficient $d_{\mu \nu}$,

$V_{d}=i d^{\mu \nu} \bar{\psi} \gamma_{\mu} \gamma_{5}\left(\partial_{v}-i e A_{\nu}\right) \psi$

include $[16,45]$. From a general perspective, the $d_{\mu \nu}$ is CPT even and therefore can only contribute to the CPT even photon coefficient $k_{F}$, however, since $d_{\mu \nu}$ is a pseudotensor, the possible LV contributions generated in the photon sector will involve even orders in $d_{\mu \nu}$, starting from a minimal term of the general form $d^{\mu \alpha} d^{\nu \beta} F_{\mu \nu} F_{\alpha \beta}$, corresponding to the generation of a $k_{F}$ term with

$\left(k_{F}\right)^{\mu \nu \alpha \beta} \sim d^{\mu \alpha} d^{\nu \beta}-d^{\nu \alpha} d^{\mu \beta}$.

A first-order term, whose only possible structure respecting observer Lorentz invariance would be like $\epsilon_{\mu \nu \alpha \rho} F^{\mu \nu} d_{\lambda}^{\alpha} F^{\lambda \rho}$, would correspond to $\left(k_{F}\right)^{\mu \nu \alpha \beta} \sim \epsilon^{\mu \nu \alpha \rho} d_{\alpha}^{\lambda}$, which does not possess the necessary symmetry properties except in the trivial case $d_{v}^{\alpha} \sim \delta_{v}^{\alpha}$,; moreover, the absence of first order in $d_{\mu \nu}$ corrections has been verified through direct calculations [16]. The second order contribution is divergent: actually, its pole part has been shown in [49] to possess the same structure as the second order in $c_{\mu \nu}$ corrections found in [47].

Despite $V_{c}$ contributing to $k_{F}$ already at the first order, and $V_{d}$ at the second order, a phenomenological analysis of these results is obscured by the fact that these corrections are divergent, and that only a particular choice of these tensors have been used in the literature to obtain explicit results.

The term proportional to $e^{\mu}$ can contribute in the quantum corrections starting at the second order: being a vector instead of a pseudo-vector, it cannot be used at first order to construct the $k_{A F}$ term, and being a CPT-odd term, it can only contribute to $k_{F}$ at second order. The same applies to $f^{\mu}$, as can be checked through straightforward calculations [49]. It turns out that both corrections have exactly the same form, amounting to divergent aether-like corrections like the ones in (8). Since the calculations in [49] where performed with an implicit regularization method, we can quote the results for the generated corrections to $k_{F}$ as

$$
\begin{aligned}
\left(k_{F}\right)^{\mu \nu \rho \sigma}= & \frac{e^{2}}{12} I_{\log }\left(m^{2}\right)\left(\eta^{\rho \mu} e^{v} e^{\sigma}\right. \\
& \left.-\eta^{\rho v} e^{\mu} e^{\sigma}-\eta^{\sigma \mu} e^{v} e^{\rho}+\eta^{\sigma v} e^{\mu} e^{\rho}\right),
\end{aligned}
$$

with the corresponding expression for $f^{\mu}$ being obtained by simply substituting $e^{\mu}$ by $f^{\mu}$, and $I_{\log }\left(m^{2}\right)$ being a loga- 
rithmically divergent expression that may be calculated in different regularization schemes.

The term proportional to $g^{\mu \nu \lambda}$ yields the finite and well defined higher-derivative contribution [50]

$\mathcal{L}_{e f f} \supset \frac{e^{2}}{24 m \pi^{2}} \mathcal{G}^{\mu v \rho \alpha \beta} A_{\alpha} \partial_{\rho} \partial_{\alpha} \partial_{\beta} A_{v}$,

$e$ being the charge and $m$ the mass of the integrated fermion, and

$$
\begin{aligned}
\mathcal{G}^{\mu \nu \rho \alpha \beta}= & g^{\mu \nu \alpha} \eta^{\rho \beta}+g^{\mu \nu \beta} \eta^{\rho \alpha}-g^{\mu \rho \alpha} \eta^{\nu \beta} \\
& -g^{\mu \rho \beta} \eta^{\nu \alpha}-g^{\rho \nu \alpha} \eta^{\mu \beta}-g^{\rho \nu \beta} \eta^{\mu \alpha} .
\end{aligned}
$$

In the SME notation, this amounts to a contribution to $\hat{k}_{A F}^{(5)}$ of the form

$\left(\hat{k}_{A F}^{(5)}\right)_{\kappa}^{\alpha \beta}=\frac{e^{2}}{24 \times 3 ! m \pi^{2}} \epsilon_{\kappa \lambda \mu \nu} \mathcal{G}^{\lambda \mu \nu \alpha \beta}$.

Despite being finite and well defined, this correction actually does not contribute to photon propagation in leading order. This can be seen by noticing that either the leading order dispersion relation [70]

$$
\left(p^{2}\right)^{2}-4\left(p^{\mu}\left(k_{A F}\right)_{\mu}\right)^{2} \approx 0
$$

or the relevant Stokes parameter [71]

$\varsigma^{3}=-p^{\mu}\left(k_{A F}\right)_{\mu} / \omega^{2}$

are modified by the combination $p^{\mu}\left(k_{A F}\right)_{\mu}$, which can be shown to vanish. Indeed, from the antisymmetry of $g^{\mu \nu \lambda}$ in the first two indices, it can be shown that

$\left(k_{A F}\right)_{\mu}=\left(\hat{k}_{A F}^{(5)}\right)_{\mu}^{\alpha \beta} p_{\alpha} p_{\beta}=6 \epsilon_{\mu \nu \rho \sigma} g^{\nu \rho \alpha} p_{\alpha} p^{\sigma}$,

and therefore $p^{\mu}\left(k_{A F}\right)_{\mu}=0$. Current limits on dimension five photon coefficients all are derived from astrophysical observations of photon propagation and, therefore, cannot be used to impose limits on $g^{\mu \nu \lambda}$ based on the induced term (23).

Finally, we note that for the particular case of completely antisymmetric $g_{\mu \nu \lambda}=\epsilon_{\mu \nu \lambda \rho} h^{\rho}$, Eq. (23) yields the finite higher-derivative CFJ-like result (6), with an appropriate multiplying factor. The finite temperature behavior of this term is discussed in [51]. In the second order in $g_{\mu \nu \lambda}$, for the same case of a completely antisymmetric $g_{\mu \nu \lambda}$, one arrives at the logarithmically divergent aether-like result (8), with $b_{\mu}$ replaced by $h_{\mu}$.

To close the discussion of the minimal part, it remains to discuss the impacts of the $H_{\mu \nu}$ term. One can naturally conclude that the lowest order contribution involving this insertion should be at least of the second order (the first-order contribution evidently vanishes by symmetry reasons), and it must be superficially finite by dimensional arguments. It is natural to expect expression of the form $H^{\mu v} H^{\alpha \beta} F_{\mu \alpha} F_{\nu \beta}$. However, explicit calculation shows that this term identically vanishes at the one-loop order [49].

\section{Non-minimal extensions of QED}

It is very natural to study quantum corrections in the minimal SME, which is proven to be a renormalizable model. In a more general perspective, the SME is an effective field theory arising in the low-energy limit of some fundamental theory at a very high energy scale $\Lambda$, therefore it depends on this characteristic energy scale, and includes non-minimal operators of mass dimension greater than four, which are, in principle, proportional to negative powers of $\Lambda[52,53]$. While the restriction to dimension three and four operators, corresponding to the minimal SME, leads to a consistent quantum field theory by itself, the general picture is certainly less clear for the non-minimal SME, since higher-dimension kinetic operators, due to the presence of higher derivatives, typically yield ghost excitations, while higher-derivative interactions are essentially non-renormalizable. So, it is not expected that consistent quantum corrections can be calculated in general, however specific terms can be shown to provide interesting results, and indeed several examples have been reported in the literature. Up to now, most of these studies focused on the leading, dimension-five, operators, with the dimension six case being discussed recently in [40] for the gauge sector, and in [54] for the spinor sector.

In this section, we will study some radiative corrections produced by non-minimal LV operators in the photon sector. We will focus mostly in results already present in the literature, which are put in a more systematic perspective. In Sect. 6, non-minimal corrections produced in the spinor will be systematically studied, and we will present new results for several dimension five coefficients.

The first non-minimal coupling to be studied at the quantum level is the dimension five magnetic one [55], involving a single $\mathrm{LV}$ vector $u_{\beta}$,

$\mathcal{V}_{1}=g u_{\beta} \bar{\psi} \gamma_{\alpha} \psi \epsilon^{\alpha \beta \gamma \delta} F_{\gamma \delta}=-\frac{1}{2}\left(a_{F}^{(5)}\right)^{\alpha \beta \gamma} \bar{\psi} \gamma_{\alpha} \psi F_{\beta \gamma}$,

where $\left(a_{F}^{(5)}\right)^{\alpha \beta \gamma}=2 g \epsilon^{\rho \alpha \beta \gamma} u_{\rho}$. There are at the moment no experimental constraints reported on these non-minimal coefficients $[8,72]$. One remarkable fact related to this vertex is that the contribution to the two-point function of the gauge field generated by two such vertices, although quadratically divergent by power counting, unexpectedly yields a finite aether-like result similar to Eq. (8), but with

$$
\begin{aligned}
\left(k_{F}\right)^{\mu \nu \alpha \beta}= & -4 C_{4} m^{2} g^{2}\left(u^{\mu} u^{\lambda} \eta^{\nu \rho}-u^{\nu} u^{\lambda} \eta^{\mu \rho}\right. \\
& \left.-u^{\mu} u^{\rho} \eta^{\lambda \rho}+u^{\nu} u^{\rho} \eta^{\mu \lambda}\right)
\end{aligned}
$$

$C_{4}$ being an ambiguous dimensionless finite constant discussed in detail in [36]. When this mechanism is considered at finite temperature, the situation becomes more involved, for example, an aether-like term involving only spatial com- 
ponents like $u_{i} u^{k} F^{i j} F_{k j}$ becomes possible [56]. The nonAbelian generalization of this calculation is possible as well. The ambiguity in the calculation of the constant $C_{4}$ in principle precludes a confident phenomenological analysis, with the objective of transferring the bounds on the generated $k_{F}$ to a bound in $a_{F}^{(5)}$, which would be a very important result.

Other corrections arising from the presence of the $\mathcal{V}_{1}$ vertex are possible. If one consider the contribution involving one $\mathcal{V}_{1}$ and one usual vertex $-e \bar{\psi} A \psi$, for example, the CFJ term proportional to the same ambiguous constant $C_{4}$ would be obtained. Calculating the higher-derivative contributions to the two-point function generated by Feynman diagrams involving either two non-minimal $\mathcal{V}_{1}$ vertices or one $\mathcal{V}_{1}$ and one usual QED vertex, with one or two minimal $V_{b}$ insertions, the result will be superficially finite, being a linear combination of the Myers-Pospelov term(13) and the higherderivative CFJ term(6), just as it occurs for the case when both vertices are minimal [39]. One of the contributions to each of these terms will be ambiguous. The complete result for the linear combination of these terms, generated by the presence of both interactions, contains (13) as one of the contributions, and looks like

$$
\begin{aligned}
\mathcal{L}_{e f f} \supset & \left(2 g^{2} C_{1}+\frac{e g}{6 \pi^{2} m^{2}}+\frac{4 e^{2}}{45 \pi^{2} m^{4}}\right) \\
& \times u^{\alpha} F_{\alpha \mu}(b \cdot \partial) u_{\beta} \epsilon^{\beta \mu \nu \lambda} F_{\nu \lambda} \\
& +\left(2 g^{2} C_{1}+\frac{e g}{6 \pi^{2} m^{2}}+\times \frac{e^{2}}{9 \pi^{2} m^{4}}\right) \\
& \times u^{2} u_{\beta} \epsilon^{\beta \mu \nu \lambda} A_{\mu} \square F_{\nu \lambda},
\end{aligned}
$$

where $C_{1}$ is the same finite and ambiguous constant involved in the generation of the CFJ term (4), as described in the previous section. Again, the remarkable property is the finiteness of this result, despite the initial power counting of the Feynman diagrams involved.

Another non-minimal, CPT odd vertex have been discussed in the literature (see f.e., [57]) in connection with axion physics,

$\mathcal{V}_{2}=v^{\beta} \bar{\psi} \gamma^{\alpha} \psi F_{\alpha \beta}$,

which is also of the same form as Eq. (29), but with

$\left(a_{F}^{(5)}\right)^{\alpha \beta \gamma}=-\left(v^{\beta} \eta^{\gamma \alpha}-v^{\alpha} \eta^{\gamma \beta}\right)$.

Notice that, here, $v^{\beta}$ has dimension of inverse of mass. It has been shown in [57] that a triangle graph similar to that one studied in [24], but with one external field $e A_{\alpha}$ replaced by the $\mathcal{V}_{2}$ vertex and the insertion $\not b \gamma_{5}$ replaced by $\vartheta \not l \gamma_{5}$, with $\vartheta=\vartheta(x)$ being the axion field, will generate in the effective action the usual coupling between the photon and an axion-like-particle, i.e.,

$$
\begin{gathered}
\mathcal{L}_{e f f} \supset C_{1} e \epsilon^{\mu \nu \alpha \beta} b_{\alpha} u^{\rho} \vartheta F_{\mu \nu} F_{\rho \beta} \\
=2 C_{1} e g(u \cdot b) \vartheta(\vec{E} \cdot \vec{B}),
\end{gathered}
$$

where $C_{1}$ is the same ambiguous constant defined in (4). In obtaining this result, it was assumed that the integrated fermion $\psi$ is very massive, so that it makes sense to extract from the relevant integrals only the dominant results when its mass is very large compared to any other scale (so it is sufficient to keep the first term in a derivative expansion for $\vartheta(x)$ ). Interestingly enough, this LV mechanism yields an isotropic correction that exactly mimics the standard axionphoton coupling, which is relevant for many experimental searches for axion-like-particles [58]. Despite being finite, this calculation suffers from the same sort of ambiguities present in the generation of the CFJ term. The possibility of a phenomenological relevance of the generated term (34) was hinted in [57], however, a proper examination of the ambiguity in this correction is still missing. Finally, as a comment, we note that if we replace the magnetic coupling in (29) by the one in (32) within the study of the aether term carried out in the paper [55], in the four-dimensional case, we will also obtain the aether term with the same ambiguous multiplier $C_{4}$ defined in (30).

The interaction vertex in (32) was further studied in a series of articles devoted to its quantum effects in the photon sector. The groundwork was developed in [59], considering the functional determinant

$S_{\text {eff }} \supset i \operatorname{Tr} \ln \left(i \not \partial-e \not A-m-\gamma^{\alpha} F_{\alpha \beta} v^{\beta}\right)$,

which was calculated using the zeta function method, and the result was expressed in a power series of the electromagnetic field strength. Wave propagation was studied with the dominant LV corrections that are generated in the photon sectors, as well as the leading non-linear corrections, i.e.,

$S_{\text {eff }} \supset \int d^{4} x\left(\mathcal{L}_{F^{4}}+\frac{g}{12 \pi^{2}} \ln \left(\frac{M^{2}}{\mu^{2}}\right) v_{\alpha} F_{\mu \nu} \partial^{\mu} F^{\nu \alpha}\right)$,

where $\mathcal{L}_{F^{4}}$ stands for the usual Lorentz invariant EulerHeisenberg Lagrangian, with the surprising result that the LV background decouples from wave propagation in vacuum, in this approximation. It is interesting to notice that this calculation does not suffer from any ambiguities of the sort involved in the generation of the CFJ term, yet the leading $\mathrm{LV}$ correction present in the last equation is divergent, thus needing a renormalization. Also, from these results one could extract additional LV non-linear terms for the field strength, which is a topic still quite unexplored in the literature. A full classification for the LV operators in gauge field theories of arbitrary dimension, including non-linear terms, have been unveiled quite recently in [7]. 
Now we focus our attention to non-minimal CPT-even couplings. The first calculation of quantum corrections involving one of these was presented in [30], involving the dimension five vertex

$\mathcal{V}_{3}=\frac{1}{2} \kappa^{\mu \nu \lambda \rho} \bar{\psi} \sigma_{\mu \nu} \psi F_{\lambda \rho}=-\frac{1}{4} H_{F}^{(5) \mu \nu \alpha \beta} \bar{\psi} \sigma_{\mu \nu} \psi F_{\alpha \beta}$,

where $H_{F}^{(5) \mu \nu \alpha \beta}=-\frac{1}{2} \kappa^{\mu \nu \lambda \rho}$ in this case. In the QED extension with this additional vertex, radiative corrections at first and second order of the LV coefficients were presented in the literature. The dominant contributions are given by [30]

$\mathcal{L}_{e f f} \supset \frac{m e}{8 \pi^{2} \epsilon} \kappa^{\mu \nu \lambda \rho} F_{\mu \nu} F_{\lambda \rho}+$ finite,

which matches the minimal CPT-even $k_{F}$ term in the SME, contributing to its renormalization. At the second order, besides a minimal $k_{F}$ term of the form $\left(k_{F}\right)^{\mu \nu \lambda \rho} \propto$ $\kappa^{\mu \nu \alpha \beta}(\kappa)_{\alpha \beta}^{\lambda \rho}$, one also will obtain the higher-derivative terms

$$
\begin{aligned}
\mathcal{L}_{e f f} \supset & \frac{1}{\pi^{2} \epsilon}\left(C_{5} \kappa^{\mu \nu \alpha \beta} \kappa_{\alpha \beta}^{\lambda \rho} F_{\mu \nu} \square F_{\lambda \rho}\right. \\
& \left.+C_{6} \kappa^{\mu \nu \alpha \beta} \kappa_{\beta}^{\gamma \lambda \rho} F_{\mu \nu} \partial_{\alpha} \partial_{\gamma} F_{\lambda \rho}\right)+ \text { finite }
\end{aligned}
$$

where $C_{5}$ and $C_{6}$ are dimensionless numerical constants. In the two last expressions, finite parts, in the UV leading order, reproduce the same tensorial structures as the pole parts.

A natural modification of the previous example consists in introducing a pseudotensor coupling [60],

$$
\begin{aligned}
\mathcal{V}_{4} & =-i g \kappa^{\mu \nu \lambda \rho} \bar{\psi} \sigma_{\mu \nu} \gamma_{5} \psi F_{\lambda \rho} \\
& =-\frac{1}{4} H_{F}^{(5) \mu \nu \alpha \beta} \bar{\psi} \sigma_{\mu \nu} \psi F_{\alpha \beta},
\end{aligned}
$$

where now $H_{F}^{(5) \mu \nu \alpha \beta}=-2 g \kappa^{\mu \nu \lambda \rho} \epsilon_{\lambda \rho}^{\alpha \beta}$. In this case, the resulting quantum corrections to the photon sector we will involve a " twisted" tensor $\bar{F}^{\mu \nu}=\kappa^{\mu \nu \lambda \rho} F_{\lambda \rho}$, together with the dual $\tilde{F}_{\mu \nu}=\frac{1}{2} \varepsilon_{\mu \nu \alpha \beta} F^{\alpha \beta}$. Again, as in the previous case, we will have contributions involving both second and higher derivatives, and they are divergent. The explicit result, as well as some consequences derived from it, have been studied in [61].

Another interaction considered in the literature, in order to generate higher-derivatives contributions in the gauge sector, is based on the Myers-Pospelov approach [38]. The idea is that additional derivatives appear in the action being contracted to some constant vector, which as a result prevents the arising of ghosts, and in the Lorentz-invariant limit the higher derivatives disappear completely. One may start by adding to the QED Lagrangian the following term,

$\mathcal{V}_{5}=\frac{1}{M^{n-1}} \bar{\psi} \gamma_{5} \psi(v \cdot D)^{n} \psi$,

with $n \geq 2$. Here $M$ is the energy scale supposed of the order of the Planck mass. This operator has mass dimensions equal to $n+3$, and have been studied in the case $n=2$ [62], where the linear combination of the higher-derivative CFJ-like term (6) and the Myers-Pospelov term (13) was generated, both being divergent. In principle, many other terms can be generated from the couplings (41), for example, it is natural to expect that the aether term can arise at least for some values of $n$.

\section{Spinor-scalar LV couplings}

The spinor-scalar LV couplings are studied in a smaller number of papers compared with the ones discussed so far, yet several interesting results have been presented in the literature. For example, the Yukawa potential was calculated in [63] considering LV just in the scalar sector, and in [64] for a LV modification of the spinor-scalar coupling of the form $\bar{\psi} G \psi \phi$, with $G$ being of the form

$G=g+i g^{\prime} \gamma_{5}+a^{\mu} \gamma_{\mu}+b^{\mu} \gamma_{5} \gamma_{\mu}+\frac{1}{2} L^{\mu \nu} \sigma_{\mu \nu}$.

Also, the one loop renormalization of a general model including fermions and scalars interacting including the aforementioned general LV Yukawa coupling was worked out in [18].

Lorentz violating Yukawa couplings have also appeared in other studies that looked into radiative corrections. The particular LV coupling

$\mathcal{Y}_{1}=a_{\mu} \bar{\psi} \gamma^{\mu} \psi \phi$,

has been used in [55] in order to generate the CPT-even aether-like term for the scalar field,

$\mathcal{L}_{\text {eff }} \supset C_{7} \phi(a \cdot \partial)^{2} \phi$,

where $C_{7}$ is a constant which diverges in four-dimensional space-time. This contributions amounts to $\left(k_{c}^{(4)}\right)^{\mu \nu}=$ $C_{7} a^{\mu} a^{\nu}$ in the SME conventions put forth in [65]. Actually, the aether term for the scalar field is the simplest Lorentz-breaking contribution for the scalar sector in the four-dimensional space-time, and in [66], it has been shown to arise also for the Lorentz-breaking spinor-scalar theory with the usual Yukawa coupling, but with Myers-Pospelovlike higher-derivative modified kinetic term for the spinor, being finite in this case.

Another interesting coupling in this sector is the pseudoscalar one,

$\mathcal{Y}_{2}=b_{\mu} \bar{\psi} \gamma^{\mu} \gamma_{5} \psi \vartheta$

where $\vartheta(x)$ is a pseudoscalar field. This vertex has been used in [57] as a part of a mechanism to generate the photon-axion term (34), as discussed in the previous section.

It is clear that, in principle, terms with more derivatives can be generated in the scalar sector as well, by the same couplings above, considering the derivative expansion of the 
two-point vertex function of the scalar, where the finiteness of these terms will be guaranteed by the renormalizability of the couplings $(43,45)$. In principle, these couplings can be used to generate the interaction terms for the Lorentz-breaking Higgs sector, although these calculations were not carried out up to now, at least in the approach we are considering. It is easy to see that since these couplings are dimensionless, the corresponding contributions to the vertices in the Higgs sector will be logarithmically divergent. Finally, it is worth mentioning the study of the Lorentz-breaking Higgs sector carried out for the scalar QED in [67], where the spontaneous symmetry breaking is considered in detail, including oneloop quantum corrections.

\section{$6 \mathrm{LV}$ contributions in the spinor sector}

The number of studies concerning the generation of LV corrections in the fermion sector of the SME is much smaller than for the scalar and the gauge sectors. At tree level, the LV extension of the spinor sector of the Standard Model was first described in the seminal papers [1,2], with the restriction of minimal (renormalizable) operators. The non-minimal fermionic sector with LV was described in [5], presenting a general parametrization for the LV coefficients, as well as discussing several aspects of these models such as dispersion relations, exact Hamiltonian and eigenstates, together with some first numerical estimations for these Lorentzbreaking parameters. More recently, the LV interaction terms involving spinors and gauge fields, with arbitrary dimension (including non-linear terms in both fields) have been systematically described in [7].

Regarding the quantum corrections, for the minimal sector, an exhaustive study of the one-loop divergent contributions to the spinor sector for the QED sector of the SME has been presented in [16], where the full one-loop renormalization of this sector was studied. In the non-minimal sector, one first result was that the CPT-odd term

$\mathcal{S}_{1}=c^{\mu \nu} \bar{\psi} \gamma_{\mu} \partial_{\nu} \psi$

was shown to arise in the non-minimal extension of the QED developed in [55] based on the non-minimal magnetic coupling (29), where $c^{\mu \nu} \sim b^{\mu} b^{\nu}$, the proportionality involving a divergent constant that needs to be renormalized. The contribution of the same structure was shown in [68] to arise also from the CPT-even coupling (37), where, however, a particular form of the $\kappa_{\mu \nu \lambda \rho}$ tensor completely described by one vector $u^{\mu}$ has been used. Again, this contribution diverges. Also, in [66], the Lorentz-breaking extension of the Yukawa model with the extra term

$$
\mathcal{S}_{2}=\bar{\psi}\left(\alpha m+g(a \cdot \partial)^{2}\right) \not h \psi
$$
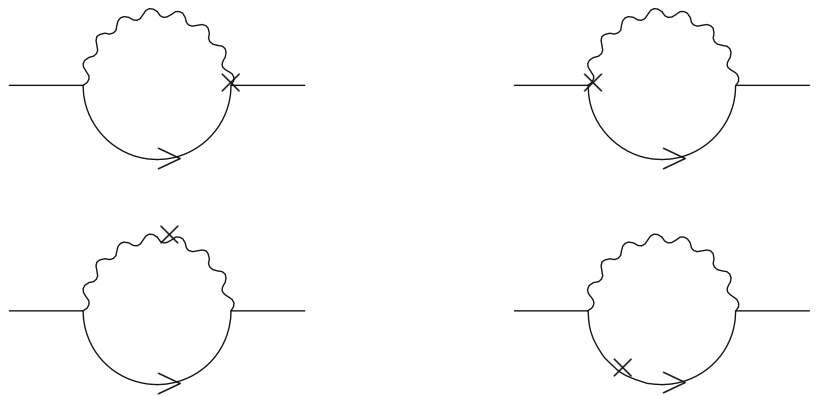

Fig. 1 One loop corrections induced from the non-minimal LV operators given in Eq. (48), where the cross indicate a LV insertion. We consider only contributions of first order in LV coefficients

$\alpha$ being a constant, has been considered and divergent quantum corrections where shown to arise for the first term in $\mathcal{S}_{1}$.

For a more systematic study of the radiative corrections involving non-minimal LV coefficients, we follow [7] and quote some of the dimension five operators involving the interaction between the fermion and the gauge field, as follows

$$
\begin{aligned}
\mathcal{L}_{\psi F}^{(5)}= & -\frac{1}{2} m_{F}^{\alpha \beta} \bar{\psi} F_{\alpha \beta} \psi-i \frac{1}{2} m_{5 F}^{\alpha \beta} \bar{\psi} \gamma_{5} F_{\alpha \beta} \psi \\
& -\frac{1}{2} a_{F}^{\mu \alpha \beta} \bar{\psi} \gamma_{\mu} F_{\alpha \beta} \psi-\frac{1}{2} b_{F}^{\mu \alpha \beta} \bar{\psi} \gamma_{5} \gamma_{\mu} F_{\alpha \beta} \psi \\
& -\frac{1}{4} H_{F}^{\mu \nu \alpha \beta} \bar{\psi} \sigma_{\mu \nu} F_{\alpha \beta} \psi+\cdots,
\end{aligned}
$$

where the dots stand for terms with second derivatives acting on $\psi$, which will be considered elsewhere (some aspects have already been discussed in [62]). We note that only the last term is CPT-even. It is interesting to mention that the contribution of the second order in $a_{F}^{\mu \alpha \beta}$ to the spinor sector have already been calculated in [55] and shown to yield an aether-like term, but the first-order contribution was not considered yet. As for the $H^{\mu \nu \alpha \beta}$, in [68] some results for a very particular form of $H^{\mu \nu \alpha \beta}$ were presented. We will now study in systematic form the radiative corrections that are induced, in the spinor sector, from these operators.

The Feynman diagrams we consider are given in Fig. 1, and the corresponding contributions, in the Feynman gauge, can be cast as

$$
\begin{aligned}
S_{1}= & \frac{1}{2} \int \frac{d^{4} p}{(2 \pi)^{4}} \bar{\psi}(-p) \\
& \times \int \frac{d^{4} k}{(2 \pi)^{4}}\left[\gamma_{\mu} \frac{1}{\not k-m} T_{\nu}[k-p] \frac{\eta^{\mu \nu}}{(k-p)^{2}}\right] \psi(p),
\end{aligned}
$$




$$
\begin{aligned}
S_{2}= & \frac{1}{2} \int \frac{d^{4} p}{(2 \pi)^{4}} \bar{\psi}(-p) \\
& \times \int \frac{d^{4} k}{(2 \pi)^{4}}\left[T_{\mu}[-(k-p)] \frac{1}{\not k-m} \gamma_{\nu} \frac{\eta^{\mu \nu}}{(k-p)^{2}}\right] \psi(p),
\end{aligned}
$$

where $T_{\mu}[k]$ is a matrix factor originating from the corresponding non-minimal vertex, depending on the incoming/outcoming momentum $k$ associated to the gauge propagator (we note that in these vertices, there is no derivatives acting on spinors). Using the Feynman representation we arrive at

$$
\begin{aligned}
S_{1}= & \frac{1}{2} \int \frac{d^{4} p}{(2 \pi)^{4}} \bar{\psi}(-p) \int \frac{d^{4} k}{(2 \pi)^{4}} \\
& \times \int_{0}^{1} d x\left[\frac{\gamma^{\mu}(k+\not k x+m) T_{\mu}[k-p(1-x)]}{\left[k^{2}+p^{2} x(1-x)-m^{2}(1-x)\right]^{2}}\right] \psi(p), \\
S_{2}= & \frac{1}{2} \int \frac{d^{4} p}{(2 \pi)^{4}} \bar{\psi}(-p) \int \frac{d^{4} k}{(2 \pi)^{4}} \\
& \times \int_{0}^{1} d x\left[\frac{T_{\mu}[-k+p(1-x)](k+\not p x+m) \gamma^{\mu}}{\left[k^{2}+p^{2} x(1-x)-m^{2}(1-x)\right]^{2}}\right] \psi(p) .
\end{aligned}
$$

It remains to calculate these expressions for various cases of $T_{\mu}$, corresponding to different operators present in Eq. (48). It should be noted that while the case of $T^{\mu}$ proportional to $a_{F}^{\mu \alpha \beta}=\epsilon^{\mu \alpha \beta \gamma} b_{\gamma}$ was considered in [55], and the secondorder contribution was found, here we obtain the contribution of first order in the Lorentz-breaking parameter. It is clear that for any Lorentz-breaking vertex, this contribution will diverge.

As a first example, we consider the vertex proportional to $H_{F}^{\mu \nu \alpha \beta}$, which corresponds to $T_{\mu}=-\frac{1}{2} H_{\alpha \beta \lambda \mu} \sigma^{\alpha \beta} \partial^{\lambda}$. In this case, the calculations will be analogous to [61], with the only difference being the presence of the $\gamma_{5}$ factor which results in the mapping $H_{\alpha \beta \lambda \mu} \rightarrow \frac{1}{2} H_{\alpha \beta}^{\sigma \rho} \epsilon_{\sigma \rho \lambda \mu}$. Therefore, we can write

$$
\begin{aligned}
\Sigma_{1}(p) & =e g H_{F}^{\mu \nu \lambda \rho} \bar{\psi}(-p) \sigma_{\mu \nu} \\
& \times \int \frac{d^{4} k}{(2 \pi)^{4}} \frac{\not k-m}{k^{2}-m^{2}} \gamma^{\alpha} \frac{1}{(p-k)^{2}}\left(p_{\lambda}-k_{\lambda}\right) \eta_{\rho \alpha} \psi(p), \\
\Sigma_{2}(p) & =-e g H_{F}^{\mu \nu \lambda \rho} \bar{\psi}(-p) \\
& \times \times i n t \frac{d^{4} k}{(2 \pi)^{4}} \gamma^{\alpha} \frac{\not k-m}{k^{2}-m^{2}} \frac{1}{(p-k)^{2}} \sigma_{\mu \nu}\left(p_{\lambda}-k_{\lambda}\right) \eta_{\rho \alpha} \psi(p),
\end{aligned}
$$

which yields, for the divergent part,

$$
\begin{aligned}
S_{1}(p) & =\frac{1}{2} \frac{e g H_{F}^{\mu \nu \lambda \rho}}{16 \pi^{2} \epsilon} \psi(-p) \sigma_{\mu \nu} \\
& \times\left(\left(\frac{m^{2}}{4}-\frac{p^{2}}{12}\right) \gamma_{\lambda} \gamma_{\rho}+\left(\frac{\not p}{6}-\frac{m}{2}\right) p_{\lambda} \gamma_{\rho}\right) \psi(p)+\cdots \\
S_{2}(p) & =\frac{1}{2} \frac{e g H_{F}^{\mu \nu \lambda \rho}}{16 \pi^{2} \epsilon} \psi(-p) \\
& \times\left(\left(\frac{m^{2}}{4}-\frac{p^{2}}{12}\right) \gamma_{\lambda} \gamma_{\rho}-\left(\frac{\not p}{6}-\frac{m}{2}\right) p_{\lambda} \gamma_{\rho}\right) \sigma_{\mu \nu} \psi(p)+\cdots
\end{aligned}
$$

So, the whole result for the first-order correction in $H_{\alpha \beta \lambda \mu}$ is found up to the second derivatives. We note that unlike in [68], these expressions do not include any restriction on the structure of $H^{\mu \nu \alpha \beta}$. We note that our result diverges, while the third- and higher-derivative orders of these contributions will be explicitly finite.

We now consider the remaining operators from (48), which can be obtained by similar calculations. We note that the radiative corrections for all of them also diverge. We note that unlike in the gauge sector [36,55], it is highly improbable to arrive at finite contributions in the spinor sector whose structure is much less restricted, which precludes the search for new constraints on LV coefficients, as we have done in Sect. 2. For example, for the $m_{F}^{\alpha \beta}$ insertion, we find

$$
\begin{aligned}
S_{1}(p)= & \frac{e g m_{F}^{\lambda \rho}}{16 \pi^{2} \epsilon} \psi(-p)\left(\left(\frac{m^{2}}{4}-\frac{p^{2}}{12}\right) \gamma_{\lambda} \gamma_{\rho}\right. \\
& \left.+\left(\frac{\not p}{6}-\frac{m}{2}\right) p_{\lambda} \gamma_{\rho}\right) \psi(p)+\cdots, \\
S_{2}(p)= & \frac{e g m_{F}^{\lambda \rho}}{16 \pi^{2} \epsilon} \psi(-p)\left(\left(\frac{m^{2}}{4}-\frac{p^{2}}{12}\right) \gamma_{\lambda} \gamma_{\rho}\right. \\
& \left.-\left(\frac{\not p}{6}-\frac{m}{2}\right) p_{\lambda} \gamma_{\rho}\right) \psi(p)+\cdots .
\end{aligned}
$$

We note that there is no restrictions on the symmetry of $m_{F}^{\alpha \beta}$, so, in principle it can be antisymmetric while up to now, only symmetric second-rank constant tensors were considered within the Lorentz-breaking context, see f.e. [47]. The presence of the antisymmetric constant tensor could be useful within the context of study of relation between Lorentz symmetry breaking and space-time noncommutativity which is typically based on use of a second-rank antisymmetric constant tensor. Similar expressions can be found for all other operators presented in (48).

Another set of dimension-five operators defined in [7] involves covariant derivatives and looks like

$$
\begin{aligned}
\mathcal{L}_{\psi D}^{(5)}= & -\frac{1}{2} m^{\alpha \beta} \bar{\psi} i D_{(\alpha} i D_{\beta)} \psi-i \frac{1}{2} m_{5}^{\alpha \beta} \bar{\psi} \gamma_{5} i D_{(\alpha} i D_{\beta)} \psi \\
& -\frac{1}{2} a^{\mu \alpha \beta} \bar{\psi} \gamma_{\mu} i D_{(\alpha} i D_{\beta)} \psi
\end{aligned}
$$


Fig. 2 Contribution to the two-point spinor function with a modified spinor propagator

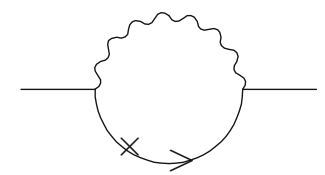

$$
\begin{aligned}
& -\frac{1}{2} b^{\mu \alpha \beta} \bar{\psi} \gamma_{5} \gamma_{\mu} i D_{(\alpha} i D_{\beta)} \psi \\
& -\frac{1}{4} H^{\mu \nu \alpha \beta} \bar{\psi} \sigma_{\mu \nu} i D_{(\alpha} i D_{\beta)} \psi+\text { h.c. }
\end{aligned}
$$

In [62], the two-point function for the $\psi$ has been obtained for the particular case of $b^{\mu \alpha \beta}=v^{\mu} v^{\alpha} v^{\beta}$. It should be noted that in this case not only new spinor-vector vertex arises, but the quadratic action of the spinor field is also modified, and we have, besides of the Feynman diagrams depicted at Fig. 1, also the graph given by Fig. 2 .

In the case of a more generic, but completely symmetric, $b^{\mu \alpha \beta}$, we can directly generalize the results obtained in [62] and get for the two first graphs given by Fig. 1,

$$
\begin{aligned}
T_{12}(k) & =-\frac{i e^{2}}{48 \pi^{2} \epsilon} \bar{\psi}(-k)\left[b_{\mu \lambda}^{\mu} \gamma^{\lambda}\left(k^{2}-3 m^{2}\right)\right. \\
& \left.-20 k^{\mu} k^{\nu} b_{\mu \nu \lambda} \gamma^{\lambda}+10 b_{\mu \lambda}^{\mu} k^{\lambda} \not k\right] \gamma_{5} \psi(k)+\ldots,
\end{aligned}
$$

and for the graph given by Fig. 2,

$$
\begin{aligned}
T_{3}(k) & =-\frac{i e^{2}}{96 \pi^{2} \epsilon} \bar{\psi}(-k)\left[\left(k^{2}-6 m^{2}\right) b_{\mu \lambda}^{\mu} \gamma^{\lambda}\right. \\
& \left.-6 b_{\mu \lambda}^{\mu} k^{\lambda} k+2 b_{\mu \nu \lambda} k^{\mu} k^{\nu} \gamma^{\lambda}\right] \gamma_{5} \psi(k) .
\end{aligned}
$$

In a similar way, this result can be generalized for other insertions. As before, all these corrections are divergent.

\section{Conclusions}

There have been extensive activities in the search for possible Lorentz violation in the last decades, which have resulted in a solid experimental program [8], as well as in a deep understanding of the theoretical questions involved in incorporating CPT and/or Lorentz breaking in the context of effective field theory. From the theoretical viewpoint, the question of quantum corrections and its effects when LV operators are considered is one of the most studied, since the seminal papers $[2,24]$. In this work, we revisit this question, filling many gaps present in the literature, when results were not written in the standard SME notation, or their phenomenological implications not fully addressed. In many instances, the generated operators are " finite but undetermined ", or divergent, however there are examples where finite, well defined corrections can be shown to exist, and these may lead to improved experimental bounds on some LV coefficients. In Sect. 2 we discussed one such case, showing how a finite quantum correction to the $k_{F}$ photon coefficient, despite being of second order in the $b^{\mu}$ coefficient that originates it, still provide a competitive constraint on some LV coefficients. This is a surprising result, since it defies the common understanding that second order LV contributions should be irrelevant for physics for being too small.

We also present some results in the spinor sector of the SME, which have not been extensively studied so far in the literature. We discuss how quantum corrections induced by dimension five LV operators, recently categorized in a systematic way in [7], can be calculated. However, in the spinor sector, we find these contributions to be divergent, on general grounds.

We conclude that still there is space for studying quantum corrections in LV theories, and that this program may even help the experimental task of constraining Lorentz violation via different experiments. Particular care should be taken to finite corrections induced in the photon sector either by nonminimal LV operators, or by higher order in the minimal ones, since the possible phenomenological implications of these have not been properly addressed in the literature.

Acknowledgements The authors would like to thank V. A. Kostelecky for discussions and interesting insights that helped us to improve our paper. This work was partially supported by Conselho Nacional de Desenvolvimento Científico e Tecnológico (CNPq) and Fundação de Amparo à Pesquisa do Estado de São Paulo (FAPESP), via the following grants: CNPq 304134/2017-1 and FAPESP 2017/13767-9 (AFF), CNPq 303783/2015-0 (AYP).

Data Availability Statement This manuscript has no associated data or the data will not be deposited. [Authors' comment: Data sharing not applicable to this article as no datasets were generated or analysed during the current study, which is of theoretical nature.]

Open Access This article is licensed under a Creative Commons Attribution 4.0 International License, which permits use, sharing, adaptation, distribution and reproduction in any medium or format, as long as you give appropriate credit to the original author(s) and the source, provide a link to the Creative Commons licence, and indicate if changes were made. The images or other third party material in this article are included in the article's Creative Commons licence, unless indicated otherwise in a credit line to the material. If material is not included in the article's Creative Commons licence and your intended use is not permitted by statutory regulation or exceeds the permitted use, you will need to obtain permission directly from the copyright holder. To view a copy of this licence, visit http://creativecomm ons.org/licenses/by/4.0/.

Funded by $\mathrm{SCOAP}^{3}$.

\section{Appendix 1: summary table}

In this Appendix, we present a table summarizing the results concerning the generation of effective operators in the photon sector of the SME, originating from the LV operators in the spinor sector, as a summary of the results discussed in Sects. 3 and 4. In formulating these tables, it is assumed that 
Table 1 Summary table of the generation of Lorentz-breaking operators in the photon sector of the SME. Notice that CPT-odd coefficients can generate CPT-even corrections at second order. Also, the axion case (pseudo-scalar field $\vartheta$ ) is not written in SME notation since the induced correction is actually Lorentz invariant, so it is not included in the traceless coefficient $k_{F}$. The columns $\mathrm{D}$ and $\mathrm{A}$ indicate whether the diagrams generating the given operator are divergent or ambiguous, respectively

\begin{tabular}{|c|c|c|c|c|c|}
\hline LV operator & CPT & Generated term & $\mathrm{D}$ & A & References \\
\hline$a_{\mu}$ & $\mathrm{O}$ & 0 & & & [49] \\
\hline \multirow[t]{3}{*}{$b_{\mu}$} & $\mathrm{O}$ & $\left(k_{A F}^{(4)}\right)_{\mu} \sim b_{\mu}$ & & $\mathrm{X}$ & {$[2,21-24]$} \\
\hline & & $\left(\hat{k}_{A F}^{(5)}\right)_{\kappa}^{\alpha \beta} \sim b_{\kappa} \eta^{\alpha \beta}$ & & & [34] \\
\hline & & $\left(k_{F}^{(4)}\right)^{\mu \nu \alpha \beta} \sim b^{\mu} b^{\alpha} \eta^{\nu \beta}+\cdots$ & & & {$[35,36]$} \\
\hline$c^{\mu \nu}$ & $\mathrm{E}$ & $\left(k_{F}^{(4)}\right)_{\mu \nu \rho \sigma} \sim c_{\mu \rho} \eta_{\nu \sigma}+\cdots$ & $\mathrm{X}$ & & [47] \\
\hline$d^{\mu \nu}$ & E & $\left(k_{F}^{(4)}\right)^{\mu \nu \rho \sigma} \sim d^{\mu \rho} d^{\nu \sigma}-d^{\mu \sigma} d^{\nu \rho}$ & $\mathrm{X}$ & & [49] \\
\hline$e^{\mu}$ & $\mathrm{O}$ & $\left(k_{F}^{(4)}\right)^{\mu \nu \rho \sigma} \sim \eta^{\rho \mu} e^{\nu} e^{\sigma}+\cdots$ & $\mathrm{X}$ & & [49] \\
\hline$f^{\mu}$ & $\mathrm{O}$ & $\left(k_{F}^{(4)}\right)^{\mu \nu \rho \sigma} \sim \eta^{\rho \mu} f^{v} f^{\sigma}+\cdots$ & $\mathrm{X}$ & & [49] \\
\hline$g^{\mu \nu \lambda}$ & $\mathrm{O}$ & $\left(\hat{k}_{A F}^{(5)}\right)_{\kappa}^{\alpha \beta} \sim \epsilon_{\kappa \lambda \mu \nu}\left(g^{\mu \nu \alpha} \eta^{\rho \beta}+\cdots\right)$ & & & {$[50]$} \\
\hline$\left(a_{F}^{(5)}\right)^{\alpha \beta \gamma}=2 g \epsilon^{\rho \alpha \beta \gamma} u_{\rho}$ & $\mathrm{O}$ & $\left(k_{F}^{(4)}\right)^{\mu \nu \alpha \beta} \sim b^{\mu} b^{\alpha} \eta^{\nu \beta}+\cdots$ & & $\mathrm{X}$ & {$[55]$} \\
\hline$\left(a_{F}^{(5)}\right)^{\alpha \beta \gamma}=-\left(u^{\beta} \eta^{\gamma \alpha}-u^{\alpha} \eta^{\gamma \beta}\right)$ & $\mathrm{O}$ & $\mathcal{L}_{e f f} \supset \epsilon^{\mu \nu \alpha \beta} b_{\alpha} u^{\rho} \vartheta F_{\mu \nu} F_{\rho \beta}$ & & $\mathrm{X}$ & [57] \\
\hline $\bar{\psi} \gamma_{5} \gamma^{\mu} \psi b_{\mu} \vartheta$ & $\mathrm{O}$ & & & & \\
\hline$H_{F}^{(5) \mu \nu \alpha \beta}=-\frac{1}{2} \kappa^{\mu \nu \lambda \rho}$ & $\mathrm{E}$ & $\left(k_{F}^{(4)}\right)_{\mu \nu \lambda \rho} \sim k_{\mu \nu \lambda \rho}$ & $\mathrm{X}$ & & {$[30]$} \\
\hline$H_{F}^{(5) \mu \nu \alpha \beta}=-2 g \kappa^{\mu \nu \lambda \rho} \epsilon_{\lambda \rho}^{\alpha \beta}$ & $\mathrm{E}$ & $\left(k_{F}^{(4)}\right)_{\mu \nu \lambda \rho} \sim k_{\mu \nu \alpha \beta} \epsilon_{\lambda \rho}^{\alpha \beta}$ & $\mathrm{X}$ & & {$[60]$} \\
\hline
\end{tabular}

the usual vertex $\sim e \bar{\psi} \gamma^{\mu} \psi A_{\mu}$ can be combined with each of the Lorentz-violating vertices involving the gauge field. For the $d^{\mu \nu}$ coefficient, the form presented in the table is derived from covariance and symmetry arguments, since no explicit results are available. For simplicity, non-Abelian generalizations are not included, but they are cited in the main text. Also, all Lorentz invariant contributions that are generated are omitted in the table, exception made for the case involving the axion field $\vartheta$, which is also the only one where the generation mechanism involves two different LV couplings (see Table 1).

\section{References}

1. D. Colladay, V.A. Kostelecky, Phys. Rev. D 55, 6760 (1997)

2. D. Colladay, V.A. Kostelecky, Phys. Rev. D 58, 116002 (1998)

3. V.A. Kostelecky, M. Mewes, Phys. Rev. D 80, 015020 (2009). arXiv:0905.0031

4. V.A. Kostelecky, M. Mewes, Phys. Rev. D 85, 096005 (2012). arXiv: 1112.6395

5. V.A. Kostelecky, M. Mewes, Phys. Rev. D 88(9), 096006 (2013). arXiv: 1308.4973

6. Y. Ding, V.A. Kostelecky, Phys. Rev. D 94, 056008 (2016). arXiv: 1608.07868

7. V.A. Kostelecky, Z. Li, Phys. Rev. D 99, 056016 (2019). arXiv: 1812.11672

8. V.A. Kostelecky, N. Russell, Rev. Mod. Phys. 83, 11 (2011). arXiv:0801.0287

9. V. A. Kostelecky, Proceedings of the 7th Meeting on CPT and Lorentz Symmetry (CPT 16): Bloomington, Indiana, USA, June 20-24, 2016, https://doi.org/10.1142/10250.
10. V.A. Kostelecky, S. Samuel, Phys. Rev. D 39, 683 (1989)

11. V.A. Kostelecky, Phys. Rev. D 69, 105009 (2004). arXiv:hep-th/0312310

12. S.M. Carroll, G.B. Field, R. Jackiw, Phys. Rev. D 41, 1231 (1990)

13. V.A. Kostelecky, M. Mewes, Phys. Rev. Lett. 87, 251304 (2001). arXiv:hep-ph/0111026

14. V.A. Kostelecky, M. Mewes, Phys. Rev. D 66, 056005 (2002). arXiv:hep-ph/0205211

15. Q.G. Bailey, V.A. Kostelecky, Phys. Rev. D 70, 076006 (2004). arXiv:hep-ph/0407252

16. V.A. Kostelecky, C.D. Lane, A.G.M. Pickering, Phys. Rev. D 65 , 056006 (2002). arXiv:hep-th/0111123

17. D. Colladay, P. McDonald, Phys. Rev. D 79, 125019 (2009). arXiv:0904.1219

18. A. Ferrero, B. Altschul, Phys. Rev. D 84, 065030 (2011). arXiv: 1104.4778

19. R. Potting, Phys. Rev. D 85, 045033 (2012). arXiv:1112.5739

20. M. Cambiaso, R. Lehnert, R. Potting, Phys. Rev. D 90, 065003 (2014). arXiv: 1401.7317

21. S.R. Coleman, S.L. Glashow, Phys. Rev. D 59, 116008 (1999). arXiv:hep-ph/9812418

22. J.M. Chung, P. Oh, Phys. Rev. D 60, 067702 (1999). arXiv:hep-th/9812132

23. A.A. Andrianov, R. Soldati, L. Sorbo, Phys. Rev. D 59, 025002 (1999). arXiv:hep-th/9806220

24. R. Jackiw, V.A. Kostelecky, Phys. Rev. Lett. 82, 3572 (1999). arXiv:hep-ph/9901358

25. R. Jackiw, Int. J. Mod. Phys. B 14, 2011 (2000). arXiv:hep-th/9903044

26. F.A. Brito, J.R. Nascimento, E. Passos, A.Y. Petrov, Phys. Lett. B 664, 112 (2008). arXiv:arXiv:0709.3090

27. O.M. Del Cima, J.M. Fonseca, D.H.T. Franco, O. Piguet, Phys. Lett. B 688, 258 (2010). arXiv:arXiv:0912.4392

28. B. Altschul, Phys. Rev. D 99, 125009 (2019). arXiv:1903.10100 [hep-th] 
29. D.L. Anderson, M. Sher, I. Turan, Phys. Rev. D 70, 016001 (2004). arXiv:hep-ph/0403116

30. R. Casana, M.M. Ferreira Jr., R.V. Maluf, F.E.P. dos Santos, Phys. Lett. B 726, 815 (2013). arXiv: 1302.2375

31. M. Gomes, J.R. Nascimento, E. Passos, A.Y. Petrov, A.J. da Silva, Phys. Rev. D 76, 047701 (2007). arXiv:0704.1104

32. S.R. Coleman, S.L. Glashow, Phys. Rev. D 59, 116008 (1999). arXiv:hep-ph/9812418

33. G. Bonneau, Nucl. Phys. B 764, 83 (2007). arXiv:hep-th/0611009

34. J. Leite, T. Mariz, W. Serafim, J. Phys. G40, 075003 (2013). arXiv: 1712.09675

35. G. Bonneau, L.C. Costa, J.L. Tomazelli, Int. J. Theor. Phys. 47, 1764 (2008). arXiv:hep-th/0510045

36. T. Mariz, J.R. Nascimento, A.Y. Petrov, B. Scarpelli, Eur. Phys. J. C 73, 2526 (2013). arXiv:1304.2256

37. V.A. Kostelecky, M. Mewes, Phys. Rev. Lett. 97, 140401 (2006). arXiv:hep-ph/0607084

38. R.C. Myers, M. Pospelov, Phys. Rev. Lett. 90, 211601 (2003). arXiv:hep-ph/0301124

39. T. Mariz, J.R. Nascimento, A.Y. Petrov, Phys. Rev. D 85, 125003 (2012). arXiv:1111.0198

40. R. Casana, M.M. Ferreira, L. Lisboa-Santos, F.E.P. dos Santos, M. Schreck, Phys. Rev. D 97, 115043 (2018). arXiv:1802.07890

41. B. Altschul, J. Phys. A 39, 13757 (2006). arXiv:hep-th/0602235

42. D. Colladay, P. McDonald, J. Math. Phys. 43, 3554 (2002). arXiv:hep-ph/0202066

43. R. Lehnert, Phys. Rev. D 74, 125001 (2006). arXiv:hep-th/0609162

44. A.F. Ferrari, R. da Rocha, J.A. Silva-Neto, Gener. Relat. Gravit. 49, 70 (2017). arXiv: 1607.08670

45. P. Arias, H. Falomir, J. Gamboa, F. Mendez, F.A. Schaposnik, Phys. Rev. D 76, 025019 (2007). arXiv:0705.3263

46. A. Salvio, Phys. Rev. D 78, 085023 (2008). arXiv:0809.0184

47. T. Mariz, R.V. Maluf, J.R. Nascimento, A.Y. Petrov, Int. J. Mod. Phys. A 33(02), 1850018 (2018). arXiv:1604.06647

48. P. Satunin, Phys. Rev. D 97(12), 125016 (2018). arXiv:1705.07796 [hep-th]

49. A.P. Baeta Scarpelli, L.C.T. Brito, J.C.C. Felipe, J.R. Nascimento, A.Y. Petrov, EPL 123, 21001 (2018). arXiv: 1805.06256

50. T. Mariz, Phys. Rev. D 83, 045018 (2011). arXiv: 1010.5013

51. J. Leite, T. Mariz, Europhys. Lett. 99, 21003 (2012). arXiv: 1110.2127

52. H. Georgi, Ann. Rev. Nucl. Part. Sci. 43, 209 (1993)

53. I. Antoniadis, E. Dudas, D.M. Ghilencea, P. Tziveloglou, Nucl. Phys. B 808, 155 (2009). arXiv:0806.3778 [hep-ph]
54. J.R. Nascimento, A.Y. Petrov, C.M. Reyes, Phys. Rev. D 92(4), 045030 (2015). arXiv:1505.04968

55. M. Gomes, J.R. Nascimento, AYu. Petrov, A.J. da Silva, Phys. Rev. D 81, 045018 (2010). arXiv:0911.3548

56. T. Mariz, J.R. Nascimento, A.Y. Petrov, W. Serafim, Phys. Rev. D 90(4), 045015 (2014). arXiv:1406.2873

57. L.H.C. Borges, A.G. Dias, A.F. Ferrari, J.R. Nascimento, A.Y. Petrov, Phys. Rev. D 89, 045005 (2014). arXiv:1304.5484

58. P.W. Graham, I.G. Irastorza, S.K. Lamoreaux, A. Lindner, K.A. van Bibber, Ann. Rev. Nucl. Part. Sci. 65, 485 (2015). arXiv: 1602.00039 [hep-ex]

59. L.H.C. Borges, A.G. Dias, A.F. Ferrari, J.R. Nascimento, A.Y. Petrov, Phys. Lett. B 756, 332 (2016). arXiv:1601.03298

60. J.B. Araujo, R. Casana, M.M. Ferreira, Phys. Lett. B 760, 302 (2016). arXiv: 1604.03577

61. A.J.G. Carvalho, A.F. Ferrari, A.M. de Lima, J.R. Nascimento, A.Y. Petrov, Nucl. Phys. B 942, 393 (2019). arXiv:1803.04308

62. T. Mariz, J.R. Nascimento, A.Y. Petrov, C.M. Reyes, Phys. Rev. D 99, 096012 (2019). arXiv: 1804.08413

63. B. Altschul, Phys. Lett. B 639, 679 (2006). arXiv:hep-th/0605044

64. B. Altschul, Phys. Rev. D 87, 045012 (2013). arXiv: 1211.6614

65. B.R. Edwards, V.A. Kostelecky, Phys. Lett. B 786, 319 (2018). arXiv: 1809.05535

66. J.R. Nascimento, A.Y. Petrov, C.M. Reyes, Eur. Phys. J. C 78(7), 541 (2018). arXiv:1706.01466

67. L.C.T. Brito, H.G. Fargnoli, A.P. Baêta Scarpelli, Phys. Rev. D 87(12), 125023 (2013). arXiv:1304.6016 [hep-th]

68. T. Mariz, J.R. Nascimento, A.Y. Petrov, H. Belich, J. Phys. Comm. 1, 045011 (2017). arXiv: 1601.03600

69. V.A. Kostelecky, M. Mewes, Phys. Lett. B 779, 136 (2018). arXiv: 1712.10268

70. This dispersion relation can be obtained from the general results presented in [3], or by the appropriate limit of the dispersion relation presented in [50], which also includes a subleading term involving a $p^{2}\left(k_{A F}\right)^{2}$ factor

71. See section IIIC of [3]

72. It is interesting to notice that in the works mentioned by us, $u^{\beta}$ is assumed to be a mass dimension one LV coefficient, just as the minimal extended QED coefficient $b^{\beta}$ (actually, in many instances, the coupling $V_{b}$ is also considered in the calculation, and it is assumed that $u^{\beta}=b^{\beta}$ ), so that $g$ has mass dimension -2 